\title{
Appliquer l'anthropologie au domaine de la santé. Entre recherche et expertise
}

Marc-Eric Gruénais

\section{OpenEdition}

\section{Journals}

Édition électronique

URL : http://journals.openedition.org/apad/1932

ISSN : 1950-6929

Éditeur

LIT Verlag

Édition imprimée

Date de publication : 1 décembre 1994

\section{Référence électronique}

Marc-Eric Gruénais, « Appliquer l'anthropologie au domaine de la santé. Entre recherche et expertise », Bulletin de l'APAD [En ligne], 8 | 1994, mis en ligne le 23 novembre 2007, consulté le 07 septembre 2020. URL : http://journals.openedition.org/apad/1932

Ce document a été généré automatiquement le 7 septembre 2020.

Bulletin de l'APAD 


\title{
Appliquer l'anthropologie au domaine de la santé. Entre recherche et expertise
}

\author{
Marc-Eric Gruénais
}

1 Dans le domaine de la santé, comme dans les autres domaines, la démarche des anthropologues apparaît régulièrement inadaptée aux yeux des responsables de programme de développement: manque de rigueur et d'objectivité de la démarche, analyses inutilement compliquées, temps trop long requis par l'enquête anthropologique, résultats par trop critiques, etc. Pourtant, si les différends entre le demandeur (médecin) et l'anthropologue sont parfois très profonds, si la prévention à l'égard d'une expertise mise en œuvre par un anthropologue est forte, on constate également que la demande d'anthropologie émanant des milieux médicaux est particulièrement importante. Je proposerai ici quelques éléments de réflexion sur ce qui ressemble souvent à un projet d'une anthropologie médicale sans anthropologue.

L'absence de dialogue

2 Depuis les années 1970 en France, la maladie, la santé sont devenues des champs privilégiés des recherches sociologiques et anthropologiques, notamment sous l'impulsion de Cl. Herzlich et M. Augé. Or, la sociologie et surtout l'anthropologie ont construit leur approche de la maladie et de la santé indépendamment des préoccupations des milieux médicaux, même si, selon S.R. Whyte (1989), on peut penser que le développement de l'intérêt des anthropologues pour ces thèmes participe d'un mouvement général de médicalisation des sociétés.

3 En France, les anthropologues revendiquent très explicitement cette distance entre anthropologie et demande médicale. Ainsi, par exemple, qualifier d"'anthropologique" une démarche qui s'inspirerait des sciences sociales et afficherait une vocation instrumentale pourrait avoir quelque chose d'incongru puisque, selon M. Augé, l'appellation "anthropologie médicale" (par opposition à "anthropologie de la maladie"), qui par nature qualifierait une démarche vouée à l'application, ne renverrait en fait qu'à une entreprise de circonstance: "... le terme medical anthropology, dans 
l'usage qu'en font les chercheurs américains, a surtout un intérêt en quelque sorte administratif et stratégique: il s'agit de rassembler sous une même étiquette (pour faire masse, ce qui peut avoir de l'intérêt quand on veut obtenir des crédits) des recherches aux finalités intellectuelles différentes qui n'ont en commun que leur objet empirique d'occasion, à condition de définir celui-ci de façon assez lâche: l'épidémiologie, l'étude des soins délivrés en institution ('health care delivery systems'), les recherches sur les problèmes de santé et l'ethnomédecine sont ainsi présentées comme les quatre grandes parties de l'anthropologie médicale elle-même conçue comme une subdivision spécifique de l'anthropologie en général..." (Augé, $1986: 81-82)$.

4 Bien plus, la notion même d"'anthropologie médicale appliquée" n'aurait pas nécessairement un sens : "... 'l'anthropologie médicale peut-elle s'appliquer ?' Se poser cette question suppose, évidemment, que l'anthropologie médicale existe en tant que discipline cohérente, c'est-à-dire comportant un corpus de propositions à peu près vérifiable et universel. Or je constate qu'on est très loin du compte et, comme j'aime quelque fois le dire, l'anthropologie médicale est plutôt une nébuleuse, de courants de recherche, d'idées, de toutes sortes d'approches, depuis la spéculation la plus effrénée jusqu'à la recherche statistique la plus rigoureuse; depuis la psychanalyse, jusqu'à l'épidémiologie, et ainsi de suite... [or] on ne peut appliquer que quelque chose qui est déjà défini et qui dispose d'un corpus de propositions générales" (Zempléni in AMADES, 1992 : 17). Dans une certaine mesure, répondre à une demande médicale équivaudrait à renoncer à être anthropologue, puisque la demande d'anthropologie médicale appliquée procéderait d'un "souhait d'une anthropologie sensiblement différente de celle qui s'enseigne ou se pratique actuellement dans les universités et les grandes institutions de recherche... on ne peut pas faire de l'ethnologie véritable à la demande ou sur commande car la demande ou la commande introduisent d'emblée une distorsion, un rétrécissement du point de vue que l'ethnologue cherche toujours à maintenir aussi large que possible" (Zempléni, 1990 : 98-99).

Cette nécessaire dissociation a priori entre une anthropologie de la maladie et la demande médicale est sans doute une spécificité française. Aux Etats-Unis, en Grande-Bretagne, la tradition de santé publique est plus ancienne et mieux ancrée qu'en France. Dès les années 50, les grands noms de la sociologie américaine (Parsons, Merton) sont associés à des programmes de recherche hospitaliers; en Grande-Bretagne, "la naissance d'une « sociologie médicale » s'inscrit dans le contexte de l'instauration, après guerre, du National Health System" (Herzlich, 1978 : 132).

Cependant, si dans le monde anglo-saxon le lien entre anthropologie médicale et demande médicale était initialement très étroit, aujourd'hui, des anthropologues d'expression anglaise semblent se ranger aux mêmes positions que celles résumées par M. Augé et A. Zempléni ci-dessus, et nombre d'anthropologues érigent en principe l'indépendance de la construction de leurs objets de recherche par rapport à la demande médicale, comme peuvent en témoigner, par exemple, les communications des participants à un atelier sur l'anthropologie médicale en Europe organisé dans le cadre de la première conférence de l'Association européenne des anthropologues sociaux (Gruénais, 1991 a).

7 Les anthropologues semblent donc réaffirmer régulièrement la nécessité du maintien d'une distance maximale entre leur discipline et la demande médicale alors même que la médecine n'a jamais été aussi ouverte qu'aujourd'hui à la démarche 
anthropologique. En effet, pour toute une série de raisons (échec des projets de développement sanitaire, faible efficacité de la médecine curative surtout pour faire face à certaines endémies, nécessité de la prévention pour réduire les coûts, retour du "culturel"), la personne du malade (son "vécu" de la maladie, ses conditions de vie, son environnement, etc.) se profile de plus en plus nettement derrière la maladie, et la demande d'anthropologie formulée par les milieux médicaux s'accroît, comme en témoigne par exemple le nombre croissant d'interventions d'anthropologues dans des sessions de formation de médecins, d'infirmier(e)s et autres personnels paramédicaux 1 .

8 Cependant la demande, de formation ou de collaboration sur un projet précis, est nécessairement très utilitaire, voire utilitariste. Les médecins attendent des enseignements dispensés par les anthropologues des moyens pratiques d'améliorer la démarche clinique, de répondre à une question d'un malade, d'expliquer pourquoi un remède traditionnel peut avoir une efficacité ${ }^{2}$. Lorsqu'il s'agit d'intervenir sur des projets, les médecins demandent aux anthropologues d'être des "techniciens auxiliaires" (Prinz, 1991), des "scouts" (Wilson, 1978) ou des "vulgarisateurs" (Basse, 1986) ; l'anthropologue doit alors répondre au problème de traduction (linguistique et culturel) posé au médecin lorsqu'il s'agit de convaincre les individus du bien-fondé de la démarche médicale.

9 Si la demande d'anthropologie est bien réelle, il n'en reste pas moins que les propos des anthropologues sont parfois totalement ignorés. Par exemple, il est tout à fait remarquable que G. Foster, un des grands noms de l'anthropologie médicale américaine, qui fut étroitement associé aux activités de l'OMS, rédige l'introduction à un ouvrage de cette institution sur la médecine traditionnelle en soulignant la complexité de la notion de maladie dans les sociétés "traditionnelles" en invitant à adopter une attitude très prudente voire critique à l'égard des tentatives d'intégration des guérisseurs traditionnels dans les programmes de soins de santé primaires (1983), alors que la plupart des articles de ce même ouvrage traitent entre autres de tout l'intérêt qu'il y aurait à utiliser les pratiques traditionnelles dans de tels programmes. Les propos que tenaient Lévi-Strauss en 1964 sont toujours d'actualité : "Notre crainte est qu'une fois de plus, les égards témoignés aux sciences sociales et humaines, la place flatteuse qui leur est faite dans un programme d'ensemble, n'aient surtout valeur d'alibi" (Levi-Strauss, 1973 : 358).

$10 \mathrm{Au}$ bout du compte, c'est l'évitement qui finit par caractériser les relations entre anthropologues et médecins, y compris aux Etats-Unis. Si des anthropologues sont associés à des médecins dans un même programme, les seconds se méfient des premiers si l'on en croit L.J. Price (1992) qui, à propos du National Institute of Health (NIH) aux USA qui contrôle les deux tiers des fonds publics alloués à la recherche médicale, constate que les recherches en sciences sociales sont considérées comme "la part non scientifique des recherches" et les recherches qualitatives, comme le "baiser de la mort" pour le NIH.

11 À force d'incompréhension, d'évitement, de volonté de reconstruire son identité contre les représentants de l'autre discipline, on en arrive à une situation de co-existence où s'observent des décalages importants entre les préoccupations des uns et des autres. Par exemple, J. Schmitz (1991) souligne bien les divergences d'intérêt lorsqu'il écrit : "... alors même que l'hôpital ou le dispensaire devenaient à leur tour objets d'investigation pour les sociologues, les organisations internationales, et en particulier l'OMS et 
l'UNICEF, préconisaient la 'sortie' de la médecine des institutions biomédicales en revalorisant les guérisseurs métamorphosés en 'tradipraticiens', ou en formant des travailleurs para-médicaux villageois". Il faut sans doute rechercher dans la spécificité de la démarche médicale cette répulsion et cette attirance conjointes pour l'anthropologie.

De l'humanisme de la démarche biomédicale à la légitimité anthropologique

La collaboration avec des médecins a ceci de particulier qu'elle engage nécessairement le collaborateur (non médecin) dans une perspective d'application, même lorsqu'il s'agit de participer à un projet de recherche. En effet, le médecin justifie sa pratique, quelle qu'elle soit, par l'action : qu'il consulte "en ville", qu'il s'adonne à la recherche, qu'il participe à un projet de développement sanitaire, le médecin pense et agit toujours en fonction de l'obligation déontologique de soigner.

Cette caractéristique de la démarche médicale qui prévaut en toutes circonstances contribue à placer les médecins dans une position délicate vis-à-vis d'une démarche de recherche. Les médecins aiment eux-mêmes à préciser que la médecine n'est pas une science mais un art, et "n'oublions pas qu'un médecin n'est pas un scientifique" rappelait J. Benoist (1989 b). Le médecin et la recherche ne font pas nécessairement bon ménage comme en témoigne ces propos de A. Fagot-Largeault $(1989: 10)$ au sujet de travaux sur les causes de mortalité : "L'entrée en médecine de l'esprit de recherche est un phénomène massif, dont la signification dépasse de loin le passage du paradigme mécaniste au paradigme probabiliste. C'est un phénomène mal admis par les médecins eux-mêmes, mal théorisé, suscitant des résistances inconscientes considérables. Il faut réaliser que le médecin qui s'interroge sur la causalité de la mort est celui qui à la fois fait des statistiques de décès sur ses propres patients, descend de l'amphithéâtre pour assister ou mettre la main à l'autopsie de leur cadavre, et dans la relation clinique a, avec eux, un rapport existentiel dont l'authenticité dépend en partie de la façon dont ils sont capables l'un et l'autre de vivre avec la mort en face". On pourrait en conclure alors que in fine le médecin ne saurait maintenir la distance nécessaire entre l'observateur et l'objet d'étude si caractéristique, sinon de la démarche scientifique, du moins de la démarche anthropologique.

Cependant cette même caractéristique qui met le médecin dans une situation où il ne peut y avoir solution de continuité entre observation scientifique et démarche thérapeutique le place également dans une position nécessairement favorable dès lors que se dessine un contexte de rapports de force entre médecine et discipline scientifique.

En effet, la médecine a également ceci de particulier qu'elle est toujours légitime au bout du compte. On peut remettre en cause un projet de développement agricole au nom de la préservation de l'écosystème, un projet de développement économique lié à des mesures d'ajustement structurel au nom du risque de la paupérisation des groupes, etc. Que cette remise en cause soit ou non suivie d'effet est une autre affaire, mais une remise en cause peut être justifiée et légitime. Mais dès qu'il s'agit d'améliorer la santé des individus, tous les arguments critiques finissent par être incongrus, voire malséants. On peut s'opposer "jusqu'au bout" à un projet d'hydraulique villageoise ; il n'est guère possible d'adopter une attitude équivalente, aussi peu adapté soit le projet, lorsqu'il s'agit de vacciner des enfants. Contribuer à endiguer la mort et la maladie, quel qu'en soit les modalités, est à l'évidence un argument d'autorité. 

dans une certaine mesure, déséquilibrée puisqu'il s'agira toujours d'être au service de la médecine ${ }^{3}$. Or la "supériorité" de la démarche médicale semble pouvoir d'autant plus s'affirmée que l'on aborde la question de l'Homme. Le discours médical est un discours humaniste : "Le discours médical n'est pas un discours sur l'homme, mais sur la maladie. Il n'en implique pas moins une certaine idée implicite sur l'homme, sur sa liberté, sur son Etre" (Clavreul, 1978). La médecine discours humaniste et non discours sur l'Homme, telle est sans doute une des raisons de l'incompréhension entre médecins et anthropologues mais qui autorise tous les débordements des premiers sur le travail des seconds. Le médecin peut considérer qu'il "chasse" sur les même terrains que l'anthropologue : "...il existe à l'intérieur des Facultés de Médecine, dans certaines des traditions du corps médical, une préoccupation qui rejoint d'une certaines façon celle des anthropologues. Elle est faite à la fois d'un fond de culture humaniste, et du souci d'une éthique centrée sur le malade. Vraisemblablement le rapport entre l'anthropologie, au sens le plus complet du terme, et les médecins pourrait se nouer à partir d'une meilleure connexion des anthropologues avec une certaine richesse culturelle qu'incarne une partie de la médecine" (Benoist in AMADES, 1992 : 102).

Il existerait donc une convergence naturelle entre médecine et anthropologie, ce qui autoriserait les médecins "à se croire 'naturellement' sociologues et anthropologues, puisqu'ils côtoient quotidiennement des problèmes humains" (D. Fassin, 1989 : 107), mais avec une légitimité plus grande : "... la pression des malades et des familles est souvent plus forte que la nécessité anthropologique ou sociologique, et la légitimité de l'acte médical l'emporte généralement sur l'urgence des sciences sociales (on se voit mal retardant un examen de malade ou un entretien avec une famille pour terminer un article...)" (Fassin, 1989 : 104).

Dans ces conditions, l'expertise anthropologique dans le domaine de la santé peut revêtir un caractère un peu particulier puisque le premier des anthropologues s'avère être le médecin qui dès lors, toujours en toute légitimité, est à même de définir une anthropologie médicale, distincte d'une anthropologie de la maladie "académique", et des méthodes anthropologiques spécifiques à sa mesure. Etre médecin autorise en effet à définir "le champ de l'anthropologie médicale" (Cathebras, 1989) ou encore à apprécier ce qu'est "le 'terrain' en anthropologie médicale" (Taverne, 1989). Cela permet également de juger ce qui est de l'anthropologie et ce qui n'en est pas. Par exemple, à propos du travail sur les systèmes de santé, un médecin déclarait : "Je me demande bien si c'est toujours de l'anthropologie médicale quand on fait de l'audit hospitalier, quand on se permet de qualifier les infirmiers, d'exclure les médecins. Si c'est toujours bien de l'anthropologie médicale quand on intervient, au nom d'un gouvernement, directement ou indirectement" (AMADES, $1992: 84$ ).

L'anthropologie des anthropologues étant inadaptée, et les médecins ayant une légitimité supérieure à celle des anthropologues professionnels à être anthropologues, ils ont donc toute latitude à créer une nouvelle discipline appelée "anthropologie médicale" qui se distingue nettement de l'anthropologie: "Pour exister comme telle, l'anthropologie appliquée au développement ne devrait-elle pas se différencier assez nettement de l'ethnologie classique...?" (Desplats, 1989: 23). Proposition volontiers reprise par certains ethnologues : "En tant que jeune ethnologue,... j'ai l'impression que lorsqu'on commence à travailler, on a effectivement des méthodes, mais les méthodes de l'ethnologie classique. On se trouve terriblement dépourvu face à des demandes 
d'anthropologie appliquée. Je ne crois pas que les méthodes de l'anthropologie classique soient transposables telles quelles à l'anthropologie appliquée. Sinon on est tout à fait coincé avec le problème du temps" (AMADES, 1992 : 95).

Pour répondre à leurs besoins d'anthropologie, les médecins élaborent alors, certes en collaboration avec certains anthropologues, des méthodes d'enquête rapide. Ainsi à propos d'une enquête réalisée dans le cadre d'une formation de personnels de santé, S. Tessier (1990: 42) écrit: "Pour des raisons pratiques l'exercice fut systématisé sous forme d'enquête socio-anthropologique rapide. Deux jours de préparation ont permis de formuler les objectifs et d'élaborer les guides d'entretien semi-structuré que les participants utilisèrent avec les familles et les professionnels. Une journée fut consacrée sur le terrain aux entretiens avec les familles et les personnels de santé en poste, en porte à porte, avec plusieurs réunions officielles ou réunions de quartiers... La synthèse de ces impressions, forcément partielles et fragmentaires en si peu de temps, permit néanmoins d'élaborer d'autres objectifs éducationnels que les objectifs classiques" ${ }^{4}$. Il existe ainsi, pour des professionnels de santé désireux de faire une étude globale d'un contexte local particulier en peu de temps, des méthodes d'évaluation anthropologique rapide (rapid assesment procedure), adaptées à des pathologies particulières (malnutrition, SIDA) ${ }^{5}$. Le médecin, muni d'une part de ses méthodes d'enquête anthropologique rapide adaptées à ses besoins et qui ont été élaborées pour être mises en œuvre par lui-même, et d'autre part de son savoir anthropologique naturel, a-t-il encore besoin de collaborer avec les anthropologues?

Vers un modus vivendi

21 On observe que les efforts de rapprochement entre les deux démarches sont principalement le fait d'anthropologues qui sont aussi médecins, et il faut reconnaître que l'anthropologie de la maladie est devenue un domaine de recherche privilégié en France également sous l'impulsion de ceux-ci. Par exemple, le premier colloque important en France sur l'anthropologie de la maladie fut coordonné par un médecin, A. Retel-Laurentin ${ }^{6} ;$ l'association AMADES qui se consacre à l'anthropologie médicale appliquée est présidée par un médecin qui est également anthropologue (J. Benoist). On pourrait aussi opposer la disparition du Bulletin d'Ethnomédecine (d'ailleurs fondé par un médecin-anthropologue, A. Epelboin) dont la publication émanait essentiellement du milieu de la recherche anthropologique, au succès d'une revue comme Sciences sociales et santé, étroitement liée au CERMES, laboratoire de recherche associé à l'INSERM. Est-ce à dire que les médecins sont plus ouverts au dialogue avec les anthropologues que l'inverse? Ces exemples illustrent surtout qu'une des solutions institutionnelles qui tend à se développer et qui permet sans doute d'éviter le divorce décrété a priori par les anthropologues, et a posteriori par les médecins entre les deux disciplines consiste à recruter, dans des organismes de recherche mais aussi dans des projets de développement, des individus disposant de la double légitimité.

Être à la fois médecin et anthropologue permet sans doute institutionnellement et statutairement d'atténuer les querelles de clocher, mais ne règle pas la question de la place de l'anthropologie dans les études sur la santé. En effet, les anthropologues, indépendamment de leur formation d'origine, s'accordent, du moins en France, pour reconnaître qu'une des spécificités de la démarche anthropologique est son approche globale (holiste, diraient certains) des réalités sociales. Or c'est sans doute cette spécificité qui gêne le plus les médecins qui, interrogeant l'anthropologue pour trouver une réponse à un problème concret, se trouve confronté à un discours sur les 
constituants de la personne, les entités religieuses, les rapports de l'homme à la nature, l'appartenance à un groupe qui est défini ou se définit comme minoritaire, mais aussi sur le système de santé et les présupposés de la démarche médicale.

Si la spécificité de la discipline anthropologique n'est pas négociable, ne convient-il pas alors d'engager la collaboration entre les deux disciplines surtout sur des thèmes de recherche qui satisferont à la fois médecins et anthropologues dans la mesure où précisément le problème de santé envisagé ne peut se résoudre qu'en adoptant une approche globale? Il existe sans aucun doute des sujets anthropologiques qui n'intéressent guère les médecins : les modalités du bouclement des cycles d'alliance dans les systèmes de parenté semi-complexes, ou encore les rituels d'intronisation d'un souverain africain ne figurent vraisemblablement pas parmi les préoccupations immédiates du médecin de santé publique chargé de la mise en place d'une intervention sanitaire. Mais inversement, toutes les questions posées par les médecins ne sont peut-être pas susceptible d'intéresser l'anthropologue.

Plus précisément, tous les symptômes font l'objet de classifications, de représentations et de pratiques, et dans cette mesure peuvent constituer des thèmes de recherche pour l'anthropologue. Cependant, il est existe peut-être des pathologies qui sont meilleures "à penser en collaboration" que d'autres. Un questionnement anthropologique autour d'une pathologie bien connue, dont les termes de la relation biunivoque établie entre une cause et son effet pathogène ${ }^{7}$ est clairement identifiée, n'est peut-être pas la meilleure "entrée" pour une collaboration. Dans ce cas, l'anthropologue risque d'être en effet immédiatement entraîné sur le terrain des "résistances culturelles" qui ne deviennent alors pertinentes pour le médecin que pour autant qu'elles sont nuisibles à la santé ${ }^{8}$, et partant sur le terrain toujours insatisfaisant pour l'anthropologue de la traduction et de la médiation culturelle 9 .

En revanche, il est des pathologies plus incertaines aux yeux des médecins, pour lesquelles les facteurs "environnementaux" et "comportementaux" sont considérés comme étant aussi important que les facteurs biologiques et qui viennent sans doute contrecarrer la tendance de la démarche médicale à privilégier les modèles causaux biologique unifactoriel au détriment des modèles causaux multifactoriels ou environnementaux (Price, 1992 :131). Sans aucun doute, le sida fait partie de ces pathologies, la malnutrition également à titre d'exemple, concernant cette dernière pathologie, j'évoquerai rapidement mon expérience de collaboration avec des épidémiologistes de la dénutrition à l'occasion d'une enquête sur les retards de croissance à Brazzaville ${ }^{10}$. Après une étude de cas auprès d'une dizaine de familles, $\mathrm{j}$ 'ai pu observer que des enfants gravement malnutris appartenaient à des ménages dont le niveau socio-économique paraissait tout à fait satisfaisant, ce qui venait remettre en cause l'existence d'une relation apparemment évidente entre déficience de l'état nutritionnel et niveau de vie ${ }^{11}$. L'explication devait alors davantage être recherchée dans des ruptures de solidarité familiale qui plaçaient une mère dans l'impossibilité d'assurer une prise en charge convenable de son enfant. Lorsqu'il s'est agi de procéder à l'enquête épidémiologique, certaines questions introduites dans le questionnaire, formulées en tenant compte des études de cas, devaient permettre d'identifier des indicateurs de rupture de solidarité (éloignement généalogique de la mère par rapport au chef de ménage, statut matrimonial précis de la mère, etc.). Les traitements statistiques ont alors permis d'identifier une population d'enfants gravement malnutris pour lesquels les facteurs de risque habituels, tel qu'un facteur d'ordre physiologique 
comme le poids de l'enfant à la naissance, ou encore les facteurs ayant trait au niveau socio-économique global des ménages cessent d'être significatifs alors que les données relatives à la famille et au statut matrimonial de la mère le deviennent.

Outre la satisfaction que peut avoir l'anthropologue lorsqu'il constate que les hypothèses émises à partir d'études de cas pouvait se trouver vérifiées en partie par une étude statistique, de tels résultats peuvent avoir des conséquences non négligeables en terme d'action. Les nutritionnistes distinguent déjà entre retard de poids et retard de taille, or parmi les enfants retardés en taille, il conviendrait sans doute de distinguer entre enfants manifestant un retard de taille modéré et enfant manifestant un retard de taille important Dans le premier cas une relation peut être établie avec des facteurs de risque déjà bien connus d'ordre biologique et individuel (poids de l'enfant à la naissance, niveau scolaire de la mère, etc.) alors que dans le second interviendraient des facteurs de risque qui ressortissent davantage aux dynamiques familiales. Si les facteurs sociaux influent sur les retards de croissance sévères, des messages d'éducation ou de prévention en terme d'éducation nutritionnelle, ou de scolarisation suffisent-ils dans tous les cas? Ne convient-il pas d'adapter les programmes aux différents groupes à risque redéfinis notamment en fonction de critères sociologiques.

Certains maux, tels que la malnutrition ou le sida, impose une approche globale également de la part des médecins. Dans ce cas, la démarche anthropologique dont il est question peut être beaucoup plus satisfaisante pour tout le monde, et notamment pour l'anthropologue qui n'a pas agi en tant que spécialiste du facteur culturel mais en tant que spécialiste de l'organisation sociale. De plus, en terme de santé publique, les réflexions auxquelles peuvent amener ces résultats me semblent toucher à des réalités tout aussi importantes que de savoir comment on nomme telle pathologie chez les $\mathrm{X}$ ou les $\mathrm{Y}$, ou comment vaincre les résistances culturelles chez les Untels afin d'améliorer l'état de santé. Entre une anthropologie médicale spécifique sans anthropologues et une anthropologie sociale qui met un point $d$ 'honneur à ignorer la demande médicale, il y a peut-être place pour une voie médiane dans laquelle médecins et anthropologues cherchent à construire et délimiter ensemble des objets qui ne sauraient être appréhendés sans une approche globale.

\section{BIBLIOGRAPHIE}

AMADES 1992 Où en est l'anthropologie appliquée ? Toulouse.

Augé, M. 1986 "L'anthropologie de la maladie", L'Homme, 97-98, XXVI (1-2) : 81-90.

Basse, M.T. 1986 "Les aspects socio-culturels et économiques de la nutrition dans les pays en voie de développement", in Lemonier, D. et Ingenbleek, Y,. (eds.), Les malnutritions dans les pays du Tiers-Monde, Colloque INSERM vol. 136, Paris, Editions de l'INSERM : 633-640.

Benoist, J. 1989 (b) "Agir, répondre : où siège l'efficacité en médecine ?", in La dimension culturelle de la maladie : quelques approches, Toulouse, AMADES : 27-36. 
Clavreul, J. 1978 L'ordre médical, Paris, Seuil.

Fagot-largeault, A. 1989 Les causes de la mort. Histoire naturelle et facteurs de risque, Paris, Librairie J. Vrin.

Foster, G.M. 1983 "Introduction à l'ethnomédecine", in Médecine traditionnelle et Couverture des Soins de Santé, Genève, OMS : 17-24.

Fassin, D. 1989 "A chacun son métier", in La recherche sous conditions, Association Française des Anthropologues, Bulletin n $36: 103-109$.

Gruenais, M.E., 1991, "L'anthropologie médicale en Europe. Etat de la question et perspectives", Journal des Anthropologues, 43-44 : 205-209.

Hagenbucher-Sacripanti, F., 1981-1982, "La représentation culturelle traditionnelle de la trypanosomiase dans le Niari (République Populaire du Congo)", Cah. ORSTOM, sér. Sci. Hum., vol. XVIII, $n^{\circ} 4$ : 445-473.

Herzlich, Cl., 1978, "Le développement de la sociologie de la médecine, en France, et son contexte", in Santé, Médecine et Sociologie, Paris, CNRS.

Horton, R. 1967 "African traditionnal thought and Western science", Africa, XXXVII, $1: 50-71$ et 2 : 155-187.

Levi-Strauss, Cl. 1973 "Critères scientifiques dans les disciplines sociales et humaines" in Anthropologie structurale deux : 339-364 [réédition d'un article paru en 1964 dans la Revue internationale des sciences sociales].

Martin, D. 1990 "Note à propos de l'enseignement de l'anthropologie dans une organisation non gouvernementale médicale", Journal des Anthropologues, 42 : 67-71.

Price, L.J., 1992, "A medical anthropologist's ruminations on NIH funding", Medical anthropology quarterly, 6 (2) : 128-146.

Prinz, A 1991 "Misunderstanding between ethnologists, pharmacologists and physicians in the field of ethnopharmacology", in Fleurentin, J. et al. (ed.), Ethnopharmacologie. Sources, méthodes, objectifs, Paris, ORSTOM/Soc. Fr. d'Ethnopharmacologie : 95-99.

Raynaut, cl., 1990, "Inégalités de santé dans une ville du Niger", Agora, 13 : 67-76.

Retel-Laurentin, A. (ed.), 1983, Une anthropologie médicale en France ? Paris, Editions du CNRS [exposés et débats de la table-ronde Santé et sciences humaines, 1980].

Schmitz, J., 1991, "Les sciences et techniques appliquées au développement et l'anthropologie", Bulletin de l'APAD, $\mathrm{n}^{\circ} 2$ : 9-15.

Tessier, S. 1990 "Le diagnostic communautaire comme outil pédagogique", in Interventions sanitaires et contextes culturels, Toulouse, AMADES : 39-46.

Whyte, S.R., 1989, "Anthropological approaches to African misfortune", in JACOBSON-Widding, A. \& Westerlund, D. (eds.), Culture, experience and pluralism. Essays on African ideas of illness and healing. Acta Universitatis Upsaliensis, Uppsala studies in cultural anthropology 13, Uppsala : 289-301.

Wilson, CH.S. 1978 "Contributions or nutrition science to anthropological research", Federation Proceedings, 37 ('1) : 73-76.

Zempleni, A. 1990 "Réflexions", in Interventions sanitaires et contextes culturels, Toulouse, AMADES : 98-99. 


\section{NOTES}

1.Il est vrai que si des anthropologues interviennent fréquemment dans ces formations médicales, leurs interventions sont alors généralement de courte durée.

2.A titre d'exemple, certes un peu caricatural, citons J. Girard, coordonnateur de l'enseignement de médecine générale pour les médecins praticiens suivant une formation continue, qui s'adressait à des anthropologues dans les termes suivants : "Que faire dans les cas suivants? M'a-t-on appris à la Faculté de médecine sinon à répondre du moins à réfléchir sur ce témoin de Jéhovah qui me refuse une gamma-globuline? M'avez-vous appris à réfléchir sur l'insuline de porc que je prescris à un musulman diabétique ? M'avez-vous appris à réfléchir sur cet écolo à tout crin qui me refuse la cortisone dans sa polyarthrite rhumatoïde ?" (AMADES, $1992:$ 59-60). 3."... les médecins attendent avant tout de l'anthropologie un service visant à répondre à des questions sur les 'résistances culturelles' à des programmes médicaux" (D. Martin, 1990 : 70 ; souligné par l'auteur).

4.Les débats autour de "la bonne méthode" sont parfaitement critiquables car, comme le soulignait $\mathrm{Cl}$. Pairault, "Une méthode ne se construit pas à partir de recettes, ou plus exactement ne s'applique pas à partir de recettes ; elle se construit à partir des objectifs prévus pour la recherche, et compte tenu des moyens donnés pour cette recherche" (AMADES, 1992 : 99).

5.A propos des RAP (Rapid Assessment Procedures), on pourra se reporter un récent article de A. Desclaux., 1992, "Le 'RAP' et les méthodes anthropologiques rapides en santé publique", Cahiers Santé, 2, : 300-306.

6.Cf. A. Retel-Laurentin (ed.), Etiologie et perception de la maladie dans les sociétés modernes et traditionnelles, Paris, L'Harmattan, 1987. Il s'agit là des actes du premier colloque national d'anthropologie médicale qui s'est tenu en 1983. Ce colloque faisait notamment suite à une table ronde organisée en 1980 intitulée "Santé et Sciences humaines" et qui avait pour but explicite de créer une anthropologie médicale en France (cf. Retel-Laurentin, 1983).

7.Pour reprendre une des caractéristiques de la pensée scientifique mise en évidence par Horton (1967).

8.Citons, à titre d'exemple, ce qu'écrit F. Hagenbucher-Sacripanti (1981-1982 : 448) au sujet de la demande médicale relative à la trypanosomiase : "'Entomologistes et médecins, chercheurs et techniciens chargés du dépistage et du traitement de la trypanosomiase observent que certaines caractéristiques culturelles et attitudes sociales propres aux populations infestées contrarient leur action... «La maladie $d u$ sommeil se heurte aux croyances traditionnelles. La façon insidieuse dont se développe la trypanosomiase dans le foyer, la lente dégradation physique, psychique et intellectuelle des malades font que les trypanosomés consultent plutôt le féticheur que le médecin»".

9.Pour témoigner de toute vaine entreprise à vouloir procéder par "traduction culturelle", on pourra se reporter à l'article de R. Schumacher, "Qu'est-ce que mara? Une approche ethnomédicale au Bèlèdougou", in J. Brunet-Jailly (ed.), Se soigner au Mali, Paris, Karthala-ORSTOM, 1993 : 49-81.

10.Pour davantage de précision sur cette enquête, je renvoie le lecteur à deux articles, M.E. Gruénais, 1985, "Mariages en ville et malnutritions aiguë", Sciences Sociales et Santé, III (3-4), 57-83 ; et M.E. Gruénais, F. Delpeuch, 1992, "Du risque au développement. Approche anthropologique et épidémiologie nutritionnelle", Cahiers des Sciences Humaines, 28 (1) : 37-55. 
11.Ces observations rejoignaient les conclusions auxquelles était parvenu CI. Raynaut (1990 : 74) après une enquête sur l'état nutritionnel des enfants à Maradi (Niger) : "Le résultat le plus frappant et le plus paradoxal de l'enquête... réside dans l'impossibilité de démontrer l'existence d'une relation entre le niveau économique d'un ménage et l'état nutritionnel de ses enfants". 